\title{
Balloon-borne measurements of temperature, water vapor, ozone and aerosol backscatter at the southern slopes of the Himalayas during StratoClim 2016-2017
}

Simone Brunamonti ${ }^{1}$, Teresa Jorge ${ }^{1}$, Peter Oelsner ${ }^{2}$, Sreeharsha Hanumanthu ${ }^{3,4}$, Bhupendra B. Singh ${ }^{3}$, K. Ravi Kumar ${ }^{3,9}$, Sunil Sonbawne ${ }^{3}$, Susanne Meier ${ }^{2}$, Deepak Singh ${ }^{5}$, Frank G. Wienhold ${ }^{1}$, Bei Ping Luo ${ }^{1}$, Maxi Böttcher ${ }^{1}$, Yann Poltera ${ }^{1}$, Hannu Jauhiainen ${ }^{8}$, Rijan Kayastha ${ }^{6}$, Ruud Dirksen ${ }^{2}$, Manish Naja ${ }^{5}$, Markus Rex ${ }^{7}$, Suvarna Fadnavis ${ }^{3}$ and Thomas Peter ${ }^{1}$

${ }^{1}$ Institute for Atmospheric and Climate Science (IAC), Swiss Federal Institute of Technology (ETH), Zürich, Switzerland ${ }^{2}$ Deutscher Wetterdienst(DWD) / GCOS Reference Upper Air Network(GRUAN) Lead Center, Lindenberg, Germany

${ }^{3}$ Indian Institute of Tropical Meteorology (IITM), Pune, India

${ }^{4}$ Forschungszentrum Jülich (FZJ), Jülich, Germany

${ }^{5}$ Aryabhatta Research Institute of Observational Sciences (ARIES), Nainital, India

${ }^{6}$ Kathmandu University (KU), Dhulikhel, Nepal

${ }^{7}$ Alfred Wegener Institute (AWI) for Polar and Marine Research, Potsdam, Germany

${ }^{8}$ Vaisala Oyj, Vantaa, Finland

${ }^{9}$ Now at King Abdullah University of Science and Technology (KAUST), Thuwal, Saudi Arabia

Correspondence to: Simone Brunamonti (simone.brunamonti@env.ethz.ch)

Supplementary material

\begin{tabular}{|l|l|l|l|l|l|l|}
\hline $\begin{array}{l}\text { Sound- } \\
\text { ing ID }\end{array}$ & Date & $\begin{array}{l}\text { Time of lau- } \\
\text { nch }\end{array}$ & Radiosonde & Payload & Status / notes & $\begin{array}{l}\text { Burst } \\
\text { height }\end{array}$ \\
\hline NT001 & $\begin{array}{l}\text { Aug 02, } \\
2016\end{array}$ & $18: 59$ UT & iMet-1-RSB & $\begin{array}{l}\text { ECC / CFH / CO- } \\
\text { BALD }\end{array}$ & - & $34.4 \mathrm{~km}$ \\
\hline NT002 & $\begin{array}{l}\text { Aug 03, } \\
2016\end{array}$ & $15: 20$ UT & iMet-1-RSB & $\begin{array}{l}\text { ECC / CFH / CO- } \\
\text { BALD }\end{array}$ & - & $29.0 \mathrm{~km}$ \\
\hline NT003 & $\begin{array}{l}\text { Aug 05, } \\
2016\end{array}$ & $17: 23$ UT & $\begin{array}{l}\text { RS41-SGP/ } \\
\text { RS92-SGP }\end{array}$ & $\begin{array}{l}\text { ECC / CFH / CO- } \\
\text { BALD }\end{array}$ & Early burst & $18.6 \mathrm{~km}$ \\
\hline NT004 & $\begin{array}{l}\text { Aug 06, } \\
2016\end{array}$ & $17: 57$ UT & $\begin{array}{l}\text { RS41-SGP / } \\
\text { RS92-SGP }\end{array}$ & $\begin{array}{l}\text { ECC / CFH / CO- } \\
\text { BALD }\end{array}$ & - & $34.0 \mathrm{~km}$ \\
\hline
\end{tabular}




\begin{tabular}{|c|c|c|c|c|c|c|}
\hline NT005 & $\begin{array}{l}\text { Aug 08, } \\
2016\end{array}$ & $16: 30 \mathrm{UT}$ & $\begin{array}{l}\text { RS41-SGP / } \\
\text { RS92-SGP }\end{array}$ & $\begin{array}{l}\mathrm{ECC} / \mathrm{CFH} / \mathrm{CO}- \\
\text { BALD }\end{array}$ & - & $30.2 \mathrm{~km}$ \\
\hline NT006 & $\begin{array}{l}\text { Aug 11, } \\
2016\end{array}$ & 05:04 UT & $\begin{array}{l}\text { RS41-SGP / } \\
\text { RS92-SGP }\end{array}$ & $\mathrm{ECC} / \mathrm{CFH}$ & $\begin{array}{l}\mathrm{CFH} \text { electronics } \\
\text { failure }\end{array}$ & $32.8 \mathrm{~km}$ \\
\hline NT007 & $\begin{array}{l}\text { Aug 11, } \\
2016\end{array}$ & $18: 57 \mathrm{UT}$ & $\begin{array}{l}\text { RS41-SGP / } \\
\text { RS92-SGP }\end{array}$ & $\begin{array}{l}\text { ECC / CFH / CO- } \\
\text { BALD }\end{array}$ & $\begin{array}{l}\text { ECC failure, } \mathrm{CFH} \\
\text { contaminated }\end{array}$ & $32.5 \mathrm{~km}$ \\
\hline NT008 & $\begin{array}{l}\text { Aug 12, } \\
2016\end{array}$ & 04:57 UT & $\begin{array}{l}\text { RS41-SGP / } \\
\text { RS92-SGP }\end{array}$ & $\mathrm{ECC} / \mathrm{CFH}$ & - & $32.0 \mathrm{~km}$ \\
\hline NT009 & $\begin{array}{l}\text { Aug 12, } \\
2016\end{array}$ & $16: 20 \mathrm{UT}$ & $\begin{array}{l}\text { RS41-SGP / } \\
\text { RS92-SGP }\end{array}$ & $\begin{array}{l}\text { ECC / CFH / CO- } \\
\text { BALD }\end{array}$ & Early burst & $19.8 \mathrm{~km}$ \\
\hline NT010 & $\begin{array}{l}\text { Aug 15, } \\
2016\end{array}$ & 04:53 UT & $\begin{array}{l}\text { RS41-SGP / } \\
\text { RS92-SGP }\end{array}$ & $\mathrm{ECC} / \mathrm{CFH}$ & $\mathrm{CFH}$ contaminated & $29.2 \mathrm{~km}$ \\
\hline NT011 & $\begin{array}{l}\text { Aug 15, } \\
2016\end{array}$ & 16:53 UT & $\begin{array}{l}\text { RS41-SGP / } \\
\text { RS92-SGP }\end{array}$ & $\begin{array}{l}\text { ECC / CFH / CO- } \\
\text { BALD }\end{array}$ & $\mathrm{CFH}$ contaminated & $26.7 \mathrm{~km}$ \\
\hline NT012 & $\begin{array}{l}\text { Aug 16, } \\
2016\end{array}$ & 03:43 UT & $\begin{array}{l}\text { RS41-SGP / } \\
\text { RS92-SGP }\end{array}$ & $\mathrm{ECC} / \mathrm{CFH}$ & - & $31.0 \mathrm{~km}$ \\
\hline NT013 & $\begin{array}{l}\text { Aug 16, } \\
2016\end{array}$ & $18: 27 \mathrm{UT}$ & $\begin{array}{l}\text { RS41-SGP / } \\
\text { RS92-SGP }\end{array}$ & $\begin{array}{l}\mathrm{ECC} / \mathrm{CFH} / \mathrm{CO}- \\
\text { BALD }\end{array}$ & $\begin{array}{l}\text { Launch failure } \\
\text { (early burst) }\end{array}$ & $2.7 \mathrm{~km}$ \\
\hline NT014 & $\begin{array}{l}\text { Aug 17, } \\
2016\end{array}$ & 10:10 UT & $\begin{array}{l}\text { RS41-SGP / } \\
\text { RS92-SGP }\end{array}$ & $\mathrm{ECC} / \mathrm{CFH}$ & - & $33.0 \mathrm{~km}$ \\
\hline NT015 & $\begin{array}{l}\text { Aug 17, } \\
2016\end{array}$ & $15: 30 \mathrm{UT}$ & $\begin{array}{l}\text { RS41-SGP / } \\
\text { RS92-SGP }\end{array}$ & $\begin{array}{l}\text { ECC / CFH / CO- } \\
\text { BALD }\end{array}$ & - & $32.7 \mathrm{~km}$ \\
\hline NT016 & $\begin{array}{l}\text { Aug 18, } \\
2016\end{array}$ & 06:28 UT & $\begin{array}{l}\text { RS41-SGP / } \\
\text { RS92-SGP }\end{array}$ & $\mathrm{ECC} / \mathrm{CFH}$ & - & $29.6 \mathrm{~km}$ \\
\hline NT017 & $\begin{array}{l}\text { Aug 18, } \\
2016\end{array}$ & 16:04 UT & $\begin{array}{l}\text { RS41-SGP / } \\
\text { RS92-SGP }\end{array}$ & $\begin{array}{l}\mathrm{ECC} / \mathrm{CFH} / \mathrm{CO}- \\
\text { BALD }\end{array}$ & - & $33.9 \mathrm{~km}$ \\
\hline NT018 & $\begin{array}{l}\text { Aug 19, } \\
2016\end{array}$ & $17: 28 \mathrm{UT}$ & $\begin{array}{l}\text { RS41-SGP / } \\
\text { RS92-SGP }\end{array}$ & $\begin{array}{l}\text { ECC / CFH / CO- } \\
\text { BALD }\end{array}$ & - & $33.0 \mathrm{~km}$ \\
\hline
\end{tabular}




\begin{tabular}{|c|c|c|c|c|c|c|}
\hline NT019 & $\begin{array}{l}\text { Aug 20, } \\
2016\end{array}$ & 03:23 UT & $\begin{array}{l}\text { RS41-SGP / } \\
\text { RS92-SGP }\end{array}$ & $\mathrm{ECC} / \mathrm{CFH}$ & - & $33.4 \mathrm{~km}$ \\
\hline NT020 & $\begin{array}{l}\text { Aug 20, } \\
2016\end{array}$ & 07:19 UT & $\begin{array}{l}\text { RS41-SGP / } \\
\text { RS92-SGP }\end{array}$ & $\mathrm{CFH}$ & - & $31.2 \mathrm{~km}$ \\
\hline NT021 & $\begin{array}{l}\text { Aug 21, } \\
2016\end{array}$ & 03:35 UT & $\begin{array}{l}\text { RS41-SGP / } \\
\text { RS92-SGP }\end{array}$ & $\mathrm{ECC} / \mathrm{CFH}$ & - & $32.3 \mathrm{~km}$ \\
\hline NT022 & $\begin{array}{l}\text { Aug 21, } \\
2016\end{array}$ & 07:12 UT & $\begin{array}{l}\text { RS41-SGP / } \\
\text { RS92-SGP }\end{array}$ & None & Early burst & $18.6 \mathrm{~km}$ \\
\hline NT023 & $\begin{array}{l}\text { Aug 21, } \\
2016\end{array}$ & 15:51 UT & $\begin{array}{l}\text { RS41-SGP / } \\
\text { RS92-SGP }\end{array}$ & $\begin{array}{l}\mathrm{ECC} / \mathrm{CFH} / \mathrm{CO}- \\
\mathrm{BALD}\end{array}$ & - & $33.0 \mathrm{~km}$ \\
\hline NT024 & $\begin{array}{l}\text { Aug 22, } \\
2016\end{array}$ & 04:21 UT & $\begin{array}{l}\text { RS41-SGP / } \\
\text { RS92-SGP }\end{array}$ & ECC & ECC battery failure & $33.6 \mathrm{~km}$ \\
\hline NT025 & $\begin{array}{l}\text { Aug 23, } \\
2016\end{array}$ & $16: 33 \mathrm{UT}$ & $\begin{array}{l}\text { RS41-SGP / } \\
\text { RS92-SGP }\end{array}$ & $\begin{array}{l}\mathrm{ECC} / \mathrm{CFH} / \mathrm{CO}- \\
\mathrm{BALD}\end{array}$ & - & $32.1 \mathrm{~km}$ \\
\hline NT026 & $\begin{array}{l}\text { Aug 24, } \\
2016\end{array}$ & 06:03 UT & $\begin{array}{l}\text { RS41-SGP / } \\
\text { RS92-SGP }\end{array}$ & $\mathrm{ECC} / \mathrm{CFH}$ & - & $29.6 \mathrm{~km}$ \\
\hline NT027 & $\begin{array}{l}\text { Aug 26, } \\
2016\end{array}$ & 18:18 UT & $\begin{array}{l}\text { RS41-SGP / } \\
\text { RS92-SGP }\end{array}$ & $\begin{array}{l}\mathrm{ECC} / \mathrm{CFH} / \mathrm{CO}- \\
\mathrm{BALD}\end{array}$ & - & $33.0 \mathrm{~km}$ \\
\hline NT028 & $\begin{array}{l}\text { Aug 28, } \\
2016\end{array}$ & 16:11 UT & $\begin{array}{l}\text { RS41-SGP / } \\
\text { RS92-SGP }\end{array}$ & $\begin{array}{l}\mathrm{ECC} / \mathrm{CFH} / \mathrm{CO}- \\
\mathrm{BALD}\end{array}$ & - & $33.9 \mathrm{~km}$ \\
\hline NT029 & $\begin{array}{l}\text { Aug 30, } \\
2016\end{array}$ & 15:44 UT & $\begin{array}{l}\text { RS41-SGP / } \\
\text { RS92-SGP }\end{array}$ & $\begin{array}{l}\mathrm{ECC} / \mathrm{CFH} / \mathrm{CO}- \\
\text { BALD }\end{array}$ & CFH contaminated & $31.3 \mathrm{~km}$ \\
\hline NT030 & $\begin{array}{l}\text { Aug 31, } \\
2016\end{array}$ & 05:58 UT & $\begin{array}{l}\text { RS41-SGP / } \\
\text { RS92-SGP }\end{array}$ & $\mathrm{ECC} / \mathrm{CFH}$ & CFH contaminated & $33.0 \mathrm{~km}$ \\
\hline NT031 & $\begin{array}{l}\text { Nov } 08 \\
2016\end{array}$ & 18:50 UT & $\begin{array}{l}\text { RS41-SGP / } \\
\text { RS92-SGP }\end{array}$ & $\begin{array}{l}\text { ECC / CFH / CO- } \\
\text { BALD }\end{array}$ & $\begin{array}{l}\text { COBALD battery } \\
\text { failure }\end{array}$ & $32.7 \mathrm{~km}$ \\
\hline NT032 & $\begin{array}{l}\text { Nov } 10 \\
2016\end{array}$ & 06:56 UT & $\begin{array}{l}\text { RS41-SGP / } \\
\text { RS92-SGP }\end{array}$ & $\mathrm{ECC} / \mathrm{CFH}$ & - & $31.5 \mathrm{~km}$ \\
\hline
\end{tabular}




\begin{tabular}{|c|c|c|c|c|c|c|}
\hline NT033 & $\begin{array}{l}\text { Nov 10, } \\
2016\end{array}$ & $14: 44 \mathrm{UT}$ & $\begin{array}{l}\text { RS41-SGP / } \\
\text { RS92-SGP }\end{array}$ & $\begin{array}{l}\text { ECC / CFH / CO- } \\
\text { BALD }\end{array}$ & - & $32.4 \mathrm{~km}$ \\
\hline NT034 & $\begin{array}{l}\text { Nov } 11, \\
2016\end{array}$ & 15:37 UT & $\begin{array}{l}\text { RS41-SGP / } \\
\text { RS92-SGP }\end{array}$ & $\begin{array}{l}\mathrm{ECC} / \mathrm{CFH} / \mathrm{CO}- \\
\mathrm{BALD}\end{array}$ & - & $32.1 \mathrm{~km}$ \\
\hline NT035 & $\begin{array}{l}\text { Nov 12, } \\
2016\end{array}$ & 07:27 UT & $\begin{array}{l}\text { RS41-SGP / } \\
\text { RS92-SGP }\end{array}$ & $\mathrm{ECC} / \mathrm{CFH}$ & $\mathrm{CFH}$ contaminated & $30.1 \mathrm{~km}$ \\
\hline DK001 & $\begin{array}{l}\text { Jul 30, } \\
2017\end{array}$ & 18:27 UT & $\begin{array}{l}\text { RS41-SGP / } \\
\text { RS92-SGP }\end{array}$ & ECC / COBALD & - & $34.8 \mathrm{~km}$ \\
\hline DK002 & $\begin{array}{l}\text { Jul 31, } \\
2017\end{array}$ & $18: 40 \mathrm{UT}$ & $\begin{array}{l}\text { RS41-SGP / } \\
\text { RS92-SGP }\end{array}$ & ECC / COBALD & - & $25.5 \mathrm{~km}$ \\
\hline DK003 & $\begin{array}{l}\text { Aug 2, } \\
2017\end{array}$ & 18:48 UT & $\begin{array}{l}\text { RS41-SGP / } \\
\text { RS92-SGP }\end{array}$ & ECC & Early burst & $18.0 \mathrm{~km}$ \\
\hline DK004 & $\begin{array}{l}\text { Aug 3, } \\
2017\end{array}$ & 19:36 UT & $\begin{array}{l}\text { RS41-SGP / } \\
\text { RS92-SGP }\end{array}$ & $\begin{array}{l}\text { ECC / CFH / CO- } \\
\text { BALD }\end{array}$ & - & $32.8 \mathrm{~km}$ \\
\hline DK005 & $\begin{array}{l}\text { Aug 4, } \\
2017\end{array}$ & $18: 18 \mathrm{UT}$ & $\begin{array}{l}\text { RS41-SGP / } \\
\text { RS92-SGP }\end{array}$ & $\mathrm{ECC} / \mathrm{CFH}$ & $\begin{array}{l}\text { CFH contamin-ated, } \\
\text { early burst }\end{array}$ & $22.7 \mathrm{~km}$ \\
\hline DK006 & $\begin{array}{l}\text { Aug 5, } \\
2017\end{array}$ & 18:09 UT & $\begin{array}{l}\text { RS41-SGP / } \\
\text { RS92-SGP }\end{array}$ & $\mathrm{ECC} / \mathrm{CFH}$ & ECC failure & $35.0 \mathrm{~km}$ \\
\hline DK007 & $\begin{array}{l}\text { Aug 6, } \\
2017\end{array}$ & 04:22 UT & $\begin{array}{l}\text { RS41-SGP / } \\
\text { RS92-SGP }\end{array}$ & - & - & $33.5 \mathrm{~km}$ \\
\hline DK008 & $\begin{array}{l}\text { Aug 6, } \\
2017\end{array}$ & 07:40 UT & $\begin{array}{l}\text { RS41-SGP / } \\
\text { RS92-SGP }\end{array}$ & - & - & $34.8 \mathrm{~km}$ \\
\hline DK009 & $\begin{array}{l}\text { Aug 6, } \\
2017\end{array}$ & 18:44 UT & $\begin{array}{l}\text { RS41-SGP / } \\
\text { RS92-SGP }\end{array}$ & $\mathrm{ECC} / \mathrm{CFH}$ & $\begin{array}{l}\text { ECC failure CFH } \\
\text { contaminated }\end{array}$ & $27.8 \mathrm{~km}$ \\
\hline DK010 & $\begin{array}{l}\text { Aug } 7 \text {, } \\
2017\end{array}$ & $16: 45 \mathrm{UT}$ & $\begin{array}{l}\text { RS41-SGP / } \\
\text { RS92-SGP }\end{array}$ & $\mathrm{ECC} / \mathrm{CFH}$ & - & $34.4 \mathrm{~km}$ \\
\hline DK011 & $\begin{array}{l}\text { Aug } 8 \\
2017\end{array}$ & 04:55 UT & $\begin{array}{l}\text { RS41-SGP / } \\
\text { RS92-SGP }\end{array}$ & - & - & $26.9 \mathrm{~km}$ \\
\hline
\end{tabular}




\begin{tabular}{|c|c|c|c|c|c|c|}
\hline DK012 & $\begin{array}{l}\text { Aug 8, } \\
2017\end{array}$ & 06:49 UT & $\begin{array}{l}\text { RS41-SGP / } \\
\text { RS92-SGP }\end{array}$ & - & - & $33.8 \mathrm{~km}$ \\
\hline DK013 & $\begin{array}{l}\text { Aug 8, } \\
2017\end{array}$ & 10:20 UT & $\begin{array}{l}\text { RS41-SGP / } \\
\text { RS92-SGP }\end{array}$ & $\mathrm{ECC} / \mathrm{CFH}$ & - & $32.4 \mathrm{~km}$ \\
\hline DK014 & $\begin{array}{l}\text { Aug 9, } \\
2017\end{array}$ & 03:51 UT & $\begin{array}{l}\text { RS41-SGP / } \\
\text { RS92-SGP }\end{array}$ & - & - & $36.9 \mathrm{~km}$ \\
\hline DK015 & $\begin{array}{l}\text { Aug 9, } \\
2017\end{array}$ & 07:18 UT & $\begin{array}{l}\text { RS41-SGP / } \\
\text { RS92-SGP }\end{array}$ & - & - & $35.3 \mathrm{~km}$ \\
\hline DK016 & $\begin{array}{l}\text { Aug 9, } \\
2017\end{array}$ & 16:49 UT & $\begin{array}{l}\text { RS41-SGP / } \\
\text { RS92-SGP }\end{array}$ & $\mathrm{ECC} / \mathrm{CFH}$ & Early burst & $19.0 \mathrm{~km}$ \\
\hline DK017 & $\begin{array}{l}\text { Aug 10, } \\
2017\end{array}$ & 03:13 UT & $\begin{array}{l}\text { RS41-SGP / } \\
\text { RS92-SGP }\end{array}$ & - & - & $33.6 \mathrm{~km}$ \\
\hline DK018 & $\begin{array}{l}\text { Aug 10, } \\
2017\end{array}$ & 05:16 UT & $\begin{array}{l}\text { RS41-SGP / } \\
\text { RS92-SGP }\end{array}$ & - & - & $35.9 \mathrm{~km}$ \\
\hline DK019 & $\begin{array}{l}\text { Aug 10, } \\
2017\end{array}$ & 07:33 UT & $\begin{array}{l}\text { RS41-SGP / } \\
\text { RS92-SGP }\end{array}$ & - & - & $36.5 \mathrm{~km}$ \\
\hline DK020 & $\begin{array}{l}\text { Aug 10, } \\
2017\end{array}$ & $16: 29 \mathrm{UT}$ & $\begin{array}{l}\text { RS41-SGP / } \\
\text { RS92-SGP }\end{array}$ & $\mathrm{ECC} / \mathrm{CFH}$ & Early burst & $18.0 \mathrm{~km}$ \\
\hline DK021 & $\begin{array}{l}\text { Aug 11, } \\
2017\end{array}$ & 02:51 UT & $\begin{array}{l}\text { RS41-SGP / } \\
\text { RS92-SGP }\end{array}$ & - & - & $35.6 \mathrm{~km}$ \\
\hline DK022 & $\begin{array}{l}\text { Aug 11, } \\
2017\end{array}$ & 05:30 UT & $\begin{array}{l}\text { RS41-SGP / } \\
\text { RS92-SGP }\end{array}$ & - & - & $32.5 \mathrm{~km}$ \\
\hline DK023 & $\begin{array}{l}\text { Aug 11, } \\
2017\end{array}$ & 07:37 UT & $\begin{array}{l}\text { RS41-SGP / } \\
\text { RS92-SGP }\end{array}$ & - & - & $33.2 \mathrm{~km}$ \\
\hline DK024 & $\begin{array}{l}\text { Aug 11, } \\
2017\end{array}$ & 11:51 UT & $\begin{array}{l}\text { RS41-SGP / } \\
\text { RS92-SGP }\end{array}$ & $\mathrm{ECC} / \mathrm{CFH}$ & - & $33.5 \mathrm{~km}$ \\
\hline
\end{tabular}




\begin{tabular}{|l|l|l|l|l|l|l|}
\hline DK025 & $\begin{array}{l}\text { Aug 12, } \\
2017\end{array}$ & $05: 44$ UT & $\begin{array}{l}\text { RS41-SGP/ } \\
\text { RS92-SGP }\end{array}$ & - & $\begin{array}{l}\text { Telemetry issues, } \\
\text { sounding term- } \\
\text { inated manually }\end{array}$ & $\begin{array}{l}\text { Un- } \\
\text { known }\end{array}$ \\
\hline DK026 & $\begin{array}{l}\text { Aug 12, } \\
2017\end{array}$ & $08: 18$ UT & $\begin{array}{l}\text { RS41-SGP/ } \\
\text { RS92-SGP }\end{array}$ & - & - & $26.8 \mathrm{~km}$ \\
\hline DK027 & $\begin{array}{l}\text { Aug 12, } \\
2017\end{array}$ & $11: 36$ UT & $\begin{array}{l}\text { RS41-SGP/ } \\
\text { RS92-SGP }\end{array}$ & CFH & CFH contaminated & $31.8 \mathrm{~km}$ \\
\hline DK028 & $\begin{array}{l}\text { Aug 12, } \\
2017\end{array}$ & $16: 53$ UT & RS41-SGP & CFH & $\begin{array}{l}\text { Early burst, CFH } \\
\text { contaminated }\end{array}$ & $22.3 \mathrm{~km}$ \\
\hline
\end{tabular}

Table S1. List of all balloon soundings performed, including sounding identifier (ID), date and time of launch, radiosondes and payload used, status / notes (instrumental malfunctionings), and burst al titude. The sounding ID is composed of the station abbreviation (NT for Nainital, India; DK for Dhul ikhel, Nepal) and sounding chronological number. Exact coordinates (latitude, longitude) of the sounding stations were, in NT: $29.3554^{\circ} \mathrm{N}, 7_{9.4619}^{\circ} \mathrm{E}$; in DK: $27.6193{ }^{\circ} \mathrm{N}, \mathbf{8 5 . 5 3 8 6}^{\circ} \mathrm{E}$. 


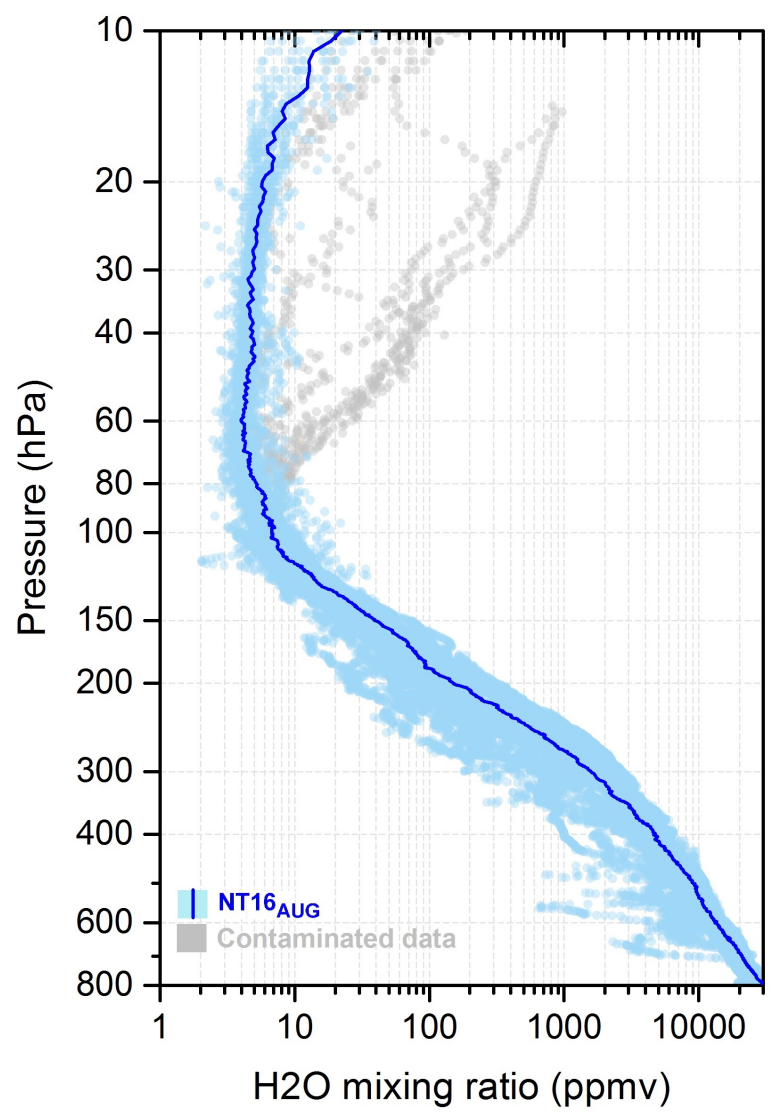

Figure S1. All measurements of $\mathrm{H}_{2} \mathrm{O}$ mixing ratio by $\mathrm{CFH}$ of the $\mathrm{NT16}_{\mathrm{AUG}}$ campaign. Blue dots: data points used for the anal ysi s. Grey dots: measurements showing CFH contamination (as described in Section 2). Blue solid line: mean profile of the data points used for the anal ysis. Contaminated measurements are detected using a threshold of $10 \mathrm{ppmv}$ in the stratosphere, and the onset of contamination is inferred as the lowest al titude where the measurement deviates significantly from the mean profile. Increased $\mathrm{H}_{2} \mathrm{O}$ mixing ratio in all measurements above $20 \mathrm{hPa}$ is likely due to outgassing from the balloon skin and the payload train (discussed in Section 3.3). 


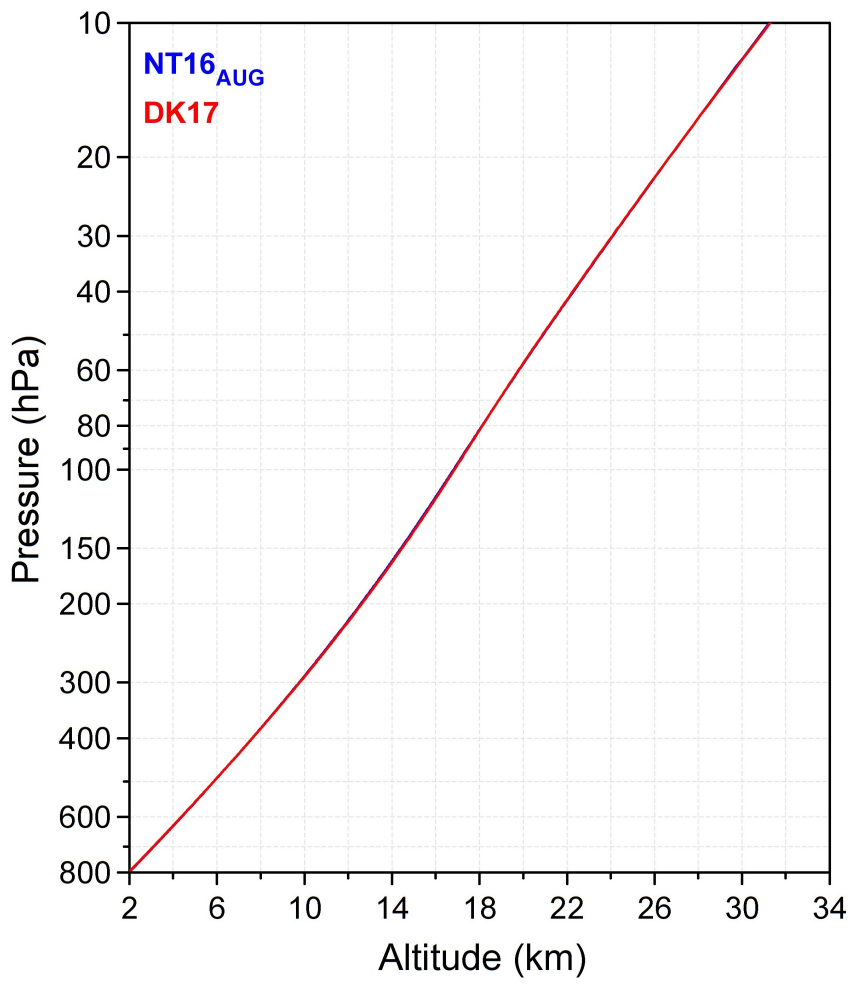

Figure S2. Mean profiles of altitude as a function of pressure from RS41-SGP measurements of the NT16 AUG $_{\text {(blue) and }}$ DK17 (red) campaigns. 

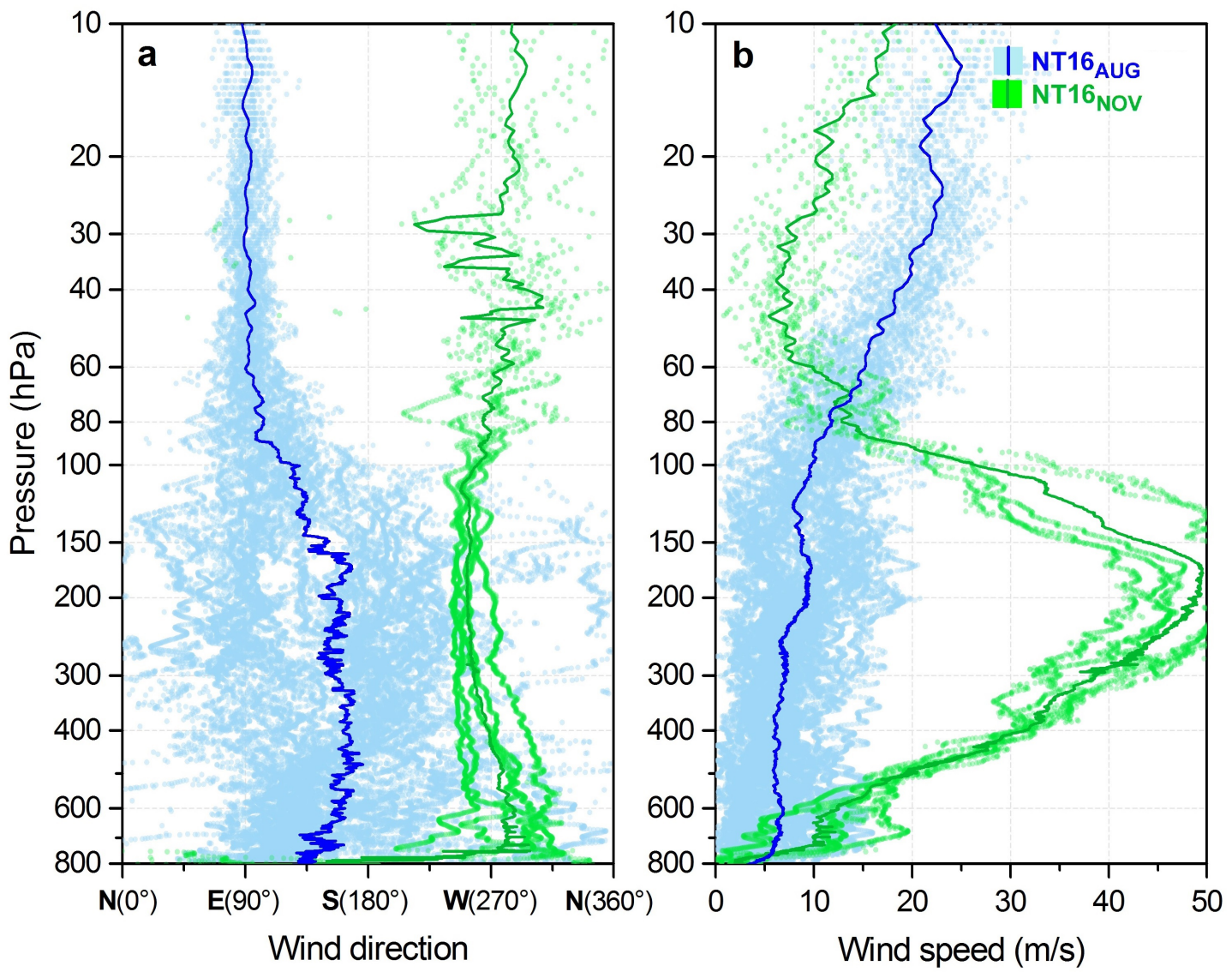

Figure S3. All measurements (dots) and mean profiles (solid lines) of wind direction (Panel a) and wind speed (b) as a

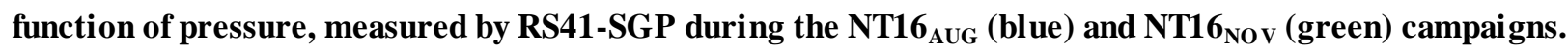




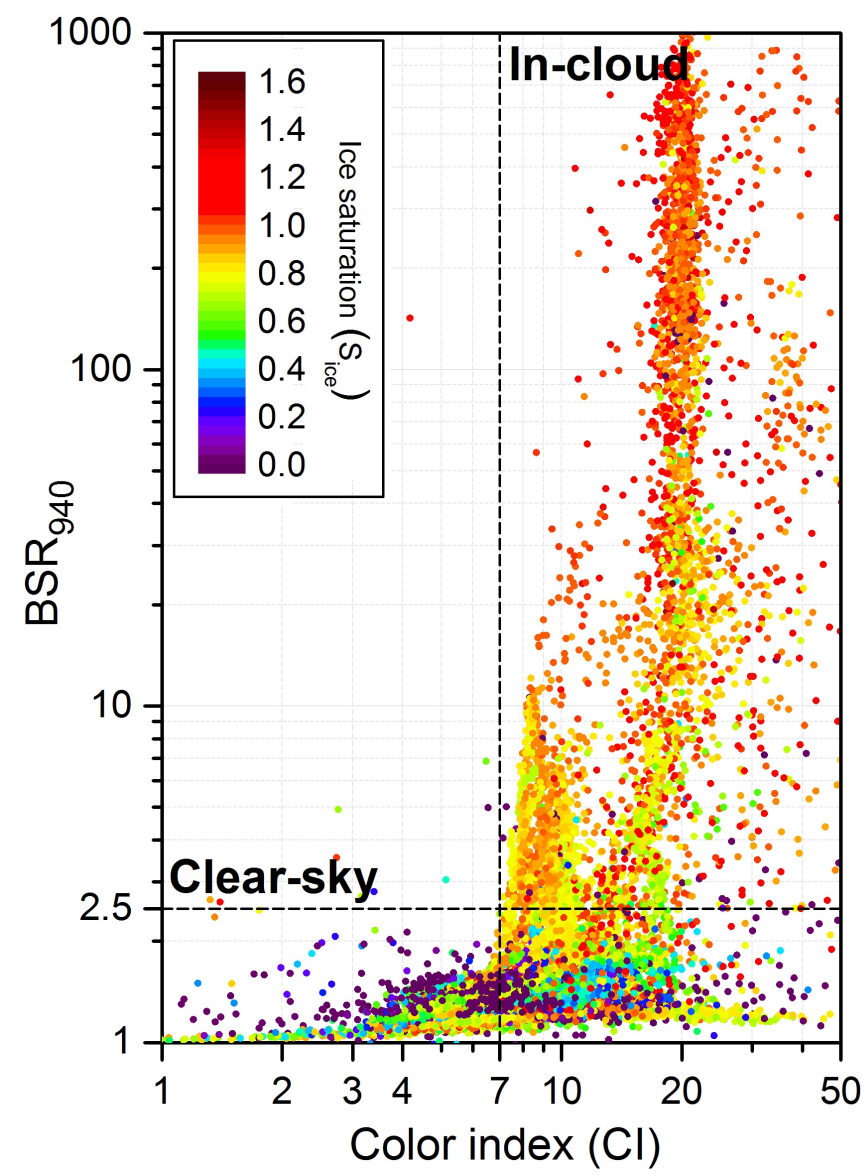

Figure S4. All measurements of the $\mathrm{NT16}_{\mathrm{AUG}}$ campaign (dots), shown as a scatter plot of BSR at $940 \mathrm{~nm}\left(\mathrm{BSR}_{940}\right)$ as a function of color index $(C I)$, color-coded with ice saturation $\left(S_{\text {ice }}\right)$. Dashed linesshow the $B S R_{940}=2.5$ and $C I=7$ isolines, which are the optical thresholds used for cloud-filtering (discussed in Section 6.2). 